\title{
The normalized Laplacian, degree-Kirchhoff index and spanning trees of graphs derived from the strong prism of linear hexagonal chain
}

Xiaocong $\mathrm{He}^{1}$

${ }^{1}$ Central South University

October 26, 2020

\begin{abstract}
Let L_n be a linear hexagonal chain with $\mathrm{n}$ hexagons. Let L^2_n be the graph obtained by the strong prism of a linear hexagonal chain with $\mathrm{n}$ hexagons, i.e. the strong product of $\mathrm{L}_{\_} \mathrm{n}$ and $\mathrm{K}_{2} 2$. In this paper, explicit expressions for degree-Kirchhoff index and number of spanning trees of $\mathrm{L}^{\wedge} 2 \_n$ are determined, respectively. Furthermore, it is interesting to find that the degree-Kirchhoff index of $L^{\wedge} 2 \_n$ is almost one eighth of its Gutman index.
\end{abstract}

\section{Hosted file}

manuscript .pdf available at https : //authorea.com/users/370314/articles/488995-the-normalizedlaplacian-degree-kirchhoff-index-and-spanning-trees-of-graphs-derived-from-the-strongprism-of-linear-hexagonal-chain
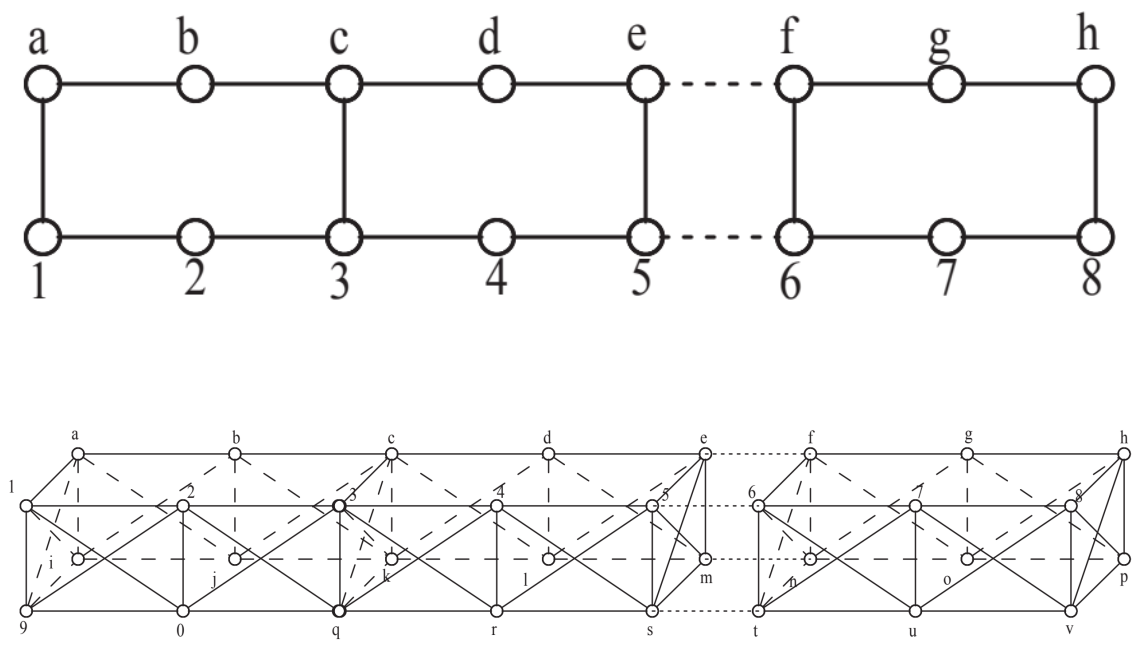